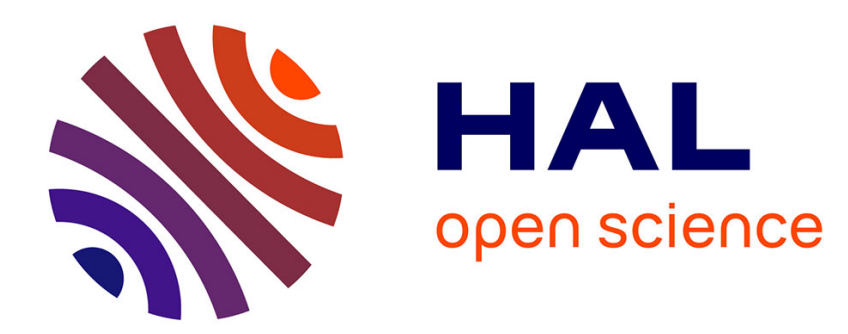

\title{
Spectroscopic Stark line profile in a non-homogeneous field quasi-static approximation
}

\author{
D. Lambert, M. Louis-Jacquet
}

\section{To cite this version:}

D. Lambert, M. Louis-Jacquet. Spectroscopic Stark line profile in a non-homogeneous field quasi-static approximation. Journal de Physique, 1985, 46 (3), pp.379-390. 10.1051/jphys:01985004603037900 . jpa-00209977

\section{HAL Id: jpa-00209977 https://hal.science/jpa-00209977}

Submitted on 1 Jan 1985

HAL is a multi-disciplinary open access archive for the deposit and dissemination of scientific research documents, whether they are published or not. The documents may come from teaching and research institutions in France or abroad, or from public or private research centers.
L'archive ouverte pluridisciplinaire HAL, est destinée au dépôt et à la diffusion de documents scientifiques de niveau recherche, publiés ou non, émanant des établissements d'enseignement et de recherche français ou étrangers, des laboratoires publics ou privés. 


\title{
Spectroscopic Stark line profile in a non-homogeneous field quasi-static approximation
}

\author{
D. Lambert and M. Louis-Jacquet \\ Commissariat à l'Energie Atomique, Centre d'Etudes de Limeil-Valenton, \\ B.P. $n^{\circ}$ 27, 94190 Villeneuve-Saint-Georges, France
}

(Reçu le 22 juin 1983, révisé le 11 octobre 1984, accepté le 7 novembre 1984)

\begin{abstract}
Résumé. - Nous précisons l'effet Stark quasi statique dans des plasmas de fusion pour des champs $F$ décrits par l'approximation de plus proche voisin $(1 \mathrm{NN})$ réduite à ses premiers termes. Les états propres correspondant sont exactement calculés en coordonnées sphériques puis convolués avec la distribution de microchamp de Hooper. Nous présentons le calcul de la raie Lyman $\gamma$ de l'argon XVIII traceur dans un mélange DT, aux densités électroniques $5 \times 10^{21} \mathrm{~cm}^{-3}$ et $7 \times 10^{22} \mathrm{~cm}^{-3}$.

Abstract. - The Stark effect in fusion plasmas is calculated for quasi-static $F$ fields corresponding to a nearest neighbour first terms approximation (1 NN). The resulting eigenstates, fully calculated in spherical coordinates, are convoluted with Hooper's microfield distribution. We present the computation of the Lyman $\gamma$ line for the tracer argon isolated in DT fuel, at electronic densities $5 \times 10^{21} \mathrm{~cm}^{-3}$ and $7 \times 10^{22} \mathrm{~cm}^{-3}$.
\end{abstract}

\section{Introduction.}

Among the various means used to diagnose dense plasmas the most interesting are surely those which provide temporally and/or spatially resolved information. An example is the spectral analysis of X-ray emission.

For laser created inertially confined plasmas with $10^{21}$ to $10^{24}$ electrons per cubic centimeter in the $200 \mathrm{eV}$ to $1 \mathrm{keV}$ temperature range; the ionization state is high enough to focus our studies on H-like ion emission. For resonance lines such as Lyman $\alpha$, the optical depth can reach 10 or more and the resulting reabsorption is so important that the line profile is mostly due to the radiative transfer along the path of the local emission. Its calculation, however, needs the initial local emission line shape and is a good motivation for studying the Stark broadening of the wings of even these reabsorbed lines. Higher upper level emission, on the other hand, provides a better investigation of the emission itself, since for the Lyman $\gamma$, for instance, the corresponding optical depth lies in the $10^{-2}-10^{-1}$ range. Then the most important broadening mechanism comes from Stark effect, except at the line center itself, and is directly related to the instantaneous local conditions, mainly electrical charge density.
This paper presents a simple computation of the argon Lyman $\gamma$ line profile for density diagnostic purposes in DT. For theoretical reasons in hydrodynamics, one should prefer experiments using a near zero argon proportion. As it is unrealistic we just consider here the DT to be preponderant, and that no argon ion is the closest to any other argon ion. The electron density range is $10^{21}$ to at least $10^{23} \mathrm{~cm}^{-3}$. At such densities, the gradient length corresponding to the screened ionic microfield can be shorter than the orbital mean radius of the optical electron. Thus the usual hypothesis of a homogeneous field is no longer valid. We present a development including the dipolar and quadrupolar terms, as the first two terms of the Taylor expansion of the microfield from the nearest perturber $(1 \mathrm{NN})$, to determine the quantum response $J(\omega, F)$ to the field $F$. The projection set is restricted to $n$-level hydrogenic states in the spherical harmonic representation. The evolution of the projections of the new eigenstates on these $|n l m\rangle$ demonstrates the importance of dipolar strength changes due to the quadrupolar term. Then the convolution of the dipolar emitting strength with Hooper's probability function $\mathfrak{T}(F)$ of the microfield provides the pure quasi-static profile. The next step includes the broadening effect of non quasi-static perturbers, which we treat by the convolution of the previous 
lines with an operator corresponding to uncorrelated electron impacts. The discussion is aimed at justifying the choice of such a quantum quasi-static response to be used with Hooper's microfield probability.

\section{Basic assumptions.}

The origin of Stark perturbation is two-fold. For a given emitter, the other ions induce a field which is screened by the cloud of all the electrons which are statistically equivalent - i.e. all the electrons but those very close to the emitter itself. To estimate the life-time of this microfield, one can divide the mean distance between ions by their classical thermal velocity. This is an under-estimated value, since statistical fluctuations exchanging two perturbers tend to maintain a constant field value. This conservative microfield life-time is then

$$
\tau>\left[\frac{4}{3} \pi N_{i}\right]^{-1 / 3}\left[\frac{2 k T_{i}}{M_{i}}\right]^{-1 / 2} .
$$

If we assume this time to be long, compared not only to the radiative period but also to the photon life-time - to be estimated after - the quantum response to this quasi-static field will represent the time averaged evolution of the real phenomena. The only differences will be caused by the shortest perturbations. So the quasi-static response $J(\omega, F)$ of the emitter determines the new eigenstates to be modified by the higher frequency part of the Stark effect, mainly electron effects.

The electrons which may not be included in the statistical quasi-static cloud are those which come in the vicinity of the emitter itself. In order not to be attracted and captured by it, they must have a sufficient kinetic energy. So their perturbation is short in time, with respect to the photon life-time but even also to the period $2 \pi / \omega$. So these perturbations, considered from the time scaling of the emitter, may be considered as collisions. We treat them as a succession of uncorrelated random impacts creating a phase confusion of the emission. This replaces a discrete quasi-static energy - or frequency - by an energy distribution of width $\hbar \Delta \omega[1]$.

To determine a correct duration-hierarchy of these phenomena, we must check that the quasi-static effective life-time of the level is much longer than the impact life-time

$$
\left[\frac{4}{3} \pi N_{i}\right]^{-1 / 3}\left[\frac{2 k T_{i}}{M_{i}}\right]^{-1 / 2} \gg \Delta \omega^{-1}
$$

We now survey the sequence of this work.

The first step is the determination of the quasistatic eigenstates from a Hamiltonian including the electrostatic perturbation $\Delta H=-q V$. Whenever the field $\mathbf{F}$ is uniform over a large domain including the optical electron range, the potential is simplified into $-q F r \cos \theta$. But, for high densities and high upper quantum ( $n=4$ for example) the mean distance from the emitting ion to its optical electron reaches the distance from itself to the nearest ion. In order to get a spatial description of the local microfield, we need a further assumption. The simplest one is that of a single perturber of charge $Z_{\mathrm{p}}$ at distance $R_{\mathrm{p}}$ from the emitting ion. With this hypothesis which requires only that all the other ions which are farther than this one keep a statistical spherical symmetry - we have

$$
\begin{gathered}
H=H_{0}-q V=H_{0}-\frac{Z_{\mathrm{p}} q^{2}}{4 \pi \varepsilon_{0}\left\|\mathbf{R}_{\mathrm{p}}-\mathbf{r}\right\|}=H_{0}+\sum_{i} V_{i} \\
F=\frac{1}{4 \pi \varepsilon_{0}} \frac{Z_{\mathrm{p}} q}{R_{\mathrm{p}}^{2}} \\
r<R_{\mathrm{p}} \Rightarrow V_{i}=-\frac{1}{R_{\mathrm{p}}} \frac{Z_{\mathrm{p}} q^{2}}{4 \pi \varepsilon_{0}}\left(\frac{r}{R_{\mathrm{p}}}\right)^{i} Y_{i}^{0}(\theta, \varphi) .
\end{gathered}
$$

The quadrupolar term provides nearly all possible couplings between the hydrogenic $|n l m\rangle$ states of the projection basis. In this paper, we keep $n$ constant that means we work in a restricted subspace only. For $n=4$ (Lyman $\gamma$ ) for instance, the single missing term would be $\langle 400|\Delta H| 430\rangle$ which requires including the octupolar term into $\Delta H$. We shall use the restricted Hamiltonian [2] :

$$
\begin{aligned}
H & =H_{0}+\Delta H_{1}+\Delta H_{2} \\
& =H_{0}-q F r \cos \theta-q F^{3 / 2} \sqrt{\frac{4 \pi \varepsilon_{0}}{Z_{\mathrm{p}} q}} r^{2} \frac{3 \cos ^{2} \theta-1}{2} .
\end{aligned}
$$

The second step deals with the emissivity of the previously calculated eigenstates. The emission power from the initial state $|i\rangle$ to the final state $|f\rangle$ is taken as the pure electrical dipolar one [3]

$$
\begin{aligned}
\omega_{\text {if }} & =\frac{E_{\mathrm{i}}-E_{\mathrm{f}}}{\hbar} \\
P\left(\omega_{\text {if }}\right) & =\rho_{\mathrm{i}} \frac{\omega_{\mathrm{if}}^{4}}{3 \pi \varepsilon_{0} c^{3}}\|\langle\mathrm{i}|\mathrm{d}| \mathrm{f}\rangle\|^{2}
\end{aligned}
$$

$\rho_{i}$ being the density in the initial state for the total configuration (the quantum state $|i\rangle$ and the surrounding conditions expressed in the value of the field and of its moments).

This expression is valid as long as $\lambda \gg\langle\mathrm{i}|r| \mathrm{i}\rangle$. In a first approximation we may factorize $\rho_{\mathrm{i}}$ as a product of the probability $\mathscr{T}(F)[4,5]$ to find $F$ by the probability $\mathfrak{T}_{F(|i\rangle)}$ to find the state $|\mathrm{i}\rangle$ among the set of eigenfunctions. As the optical electron is the single moving particle of the system, this probability obeys Fermi statistics.

For any given value of $F$ :

$$
\mathfrak{T}_{F(|\mathrm{i}\rangle)}=\frac{1}{\mathrm{e}^{\hbar \omega_{\text {if }} / k T}+1} \frac{1}{Z_{\mathrm{F}}(T)}
$$


where $Z_{\mathrm{F}}(T)$ is the partition function. There is no degeneracy factor $g_{i}$ here, since we make the summation over the new and all different eigenstates.

Then the total quasi-static emitting power becomes :

$$
\begin{aligned}
P(\omega)=\sum_{|\mathrm{i}\rangle} \int \mathrm{d} F \mathfrak{T}(F) \frac{\omega^{4}}{4 \pi \varepsilon_{0} c^{3}} \times \\
\times \mathfrak{T}_{F(|\mathrm{i}\rangle)}\|\langle\mathrm{i}|d| \mathrm{f}\rangle\|^{2} \delta\left(\omega-\omega_{\mathrm{if}}\right) .
\end{aligned}
$$

So one usually defines a so-called « line-profile » [6] as :

$$
J(\omega)=\sum_{|\mathrm{i}\rangle} \int \mathrm{d} F \mathfrak{T}(F)\|\langle\mathrm{i}|\mathrm{d}| \mathrm{f}\rangle\|^{2} \delta\left(\omega-\omega_{\mathrm{if}}\right) .
$$

The difference with the previous expression is the factor $K(\omega)$ inside the integration :

$$
K(\omega)=\frac{\omega^{4}}{4 \pi \varepsilon_{0} c^{3}} \mathfrak{T}_{F(|\mathrm{i}\rangle)}
$$

In order to make the search of new eigenstates easier, the investigation of the new eigenstates uses the projection on the subspace defined by a given value of $n$ only. Then we must restrict the broadening frequency range to the limit of confusion with other $n$, as in the Inglis-Teller like condition :

$$
\Delta \omega<\frac{Z^{2} E_{\mathrm{I}}}{\hbar} \frac{1+2 n}{n^{2}(n+1)^{2}}
$$

where $E_{\mathrm{I}}$ is the hydrogen ionization energy. For medium $Z$ elements, we are limited about $\frac{\Delta \omega}{\omega} \leqslant 3 \%$, and we checked that, within this range, the maximum variation of $K(\omega)$ is less than $10 \%$. So we present the line profile $J(\omega)$ instead of the real emission $P(\omega)$. The last step, which is the convolution of quasistatic results by electron impacts is also presented in the $J(\omega)$ form.

\section{Calculation of the new quasi-static eigenstates.}

We use the spherical harmonic functions, solutions of the unperturbed hydrogen-like Hamiltonian $H_{0}$, as a basis of representation $\{|n l m\rangle\}$. Parabolic coordinates $\left(n_{1}, n_{2}, m\right)$ have often been used [7] since they provide a direct diagonalization of the linear perturbation $\Delta H_{1}=-q F r \cos \theta$. These functions, however, are not convenient for the further terms of the Hamiltonian, coming from a more suitable description of the microfield. Instead of an approximate perturbation method solving $\Delta \mathrm{H}_{2}$ or any further term from these parabolic states solutions of $H_{0}+\Delta H_{1}$, we prefer the exact diagonalization of the complete new Hamiltonian over the complete set of hydrogen-like functions $\{|n l m\rangle\}$. Since a single perturber approximation keeps axial symmetry, the new splitting does not mix states of different magnetic projections $m$, and does not remove the degeneracy induced by $\pm m$.

We restrict our re-diagonalization to the states $\{|n l m\rangle\}, n$ being a constant (here $n=4)$. Since this is not a complete projection set, the calculation is not exact but remains valid enough inside the InglisTeller limitation. So the identification of the new eigenstates can use the previous quantum numbers $n$ and $m$. We complete this identification by the energy rank $i$ inside each $(n, m)$ class. To distinguish this notation from that of the spherical harmonics $|\mathrm{nlm}\rangle$ the representation of a new eigenstate will be $|\overline{\text { nim }}\rangle$. After separation of the hydrogenic eigenstates according to their magnetic projection, the $16 \times 16$ matrix corresponding to the $n=4$ level is factorized into a product of simpler matrices (the spin projection is of no interest here and we ignore its factor-2 complexity).

Our expression of the perturbation uses the assumption that $r \leqslant R_{\mathrm{p}}$. If we evaluate $r$ as the value of Bohr radius, $r=a_{0} n^{2} / Z$ and calculate the field corresponding to that limit, the maximal range of the calculation is then for $n^{*}=4$.

$$
\begin{aligned}
& F_{\mathrm{m}}=6.5 \times 10^{11} \mathrm{~V} \cdot \mathrm{m}^{-1} \text { for } \mathrm{XVIII}_{\text {argon in } \mathrm{D}^{+} \mathrm{T}^{+}} \\
& F_{\mathrm{m}}=1.2 \times 10^{13} \mathrm{~V} \cdot \mathrm{m}^{-1} \text { for pure } \mathrm{XVIIII}_{\text {argon }} \\
& F_{\mathrm{m}}=2.0 \times 10^{12} \mathrm{~V} \cdot \mathrm{m}^{-1} \text { for pure } \mathrm{x}_{\text {neon }} .
\end{aligned}
$$

Although this limit is physically important, we show the curves for field values up to $10^{13} \mathrm{Vm}^{-1}$, in order to complete the mathematical evolution. For these last values, however, the physical meaning of the calculation is uncertain since the electron is correlated to both nuclei.

A $4 \times 4$ matrix deals with initial states $|400\rangle$, $|410\rangle,|420\rangle$ and $|430\rangle$ corresponding to $m=0$. The energy solutions are non-degenerate and presented in figure 1 versus the value of the field $F$. In reduced

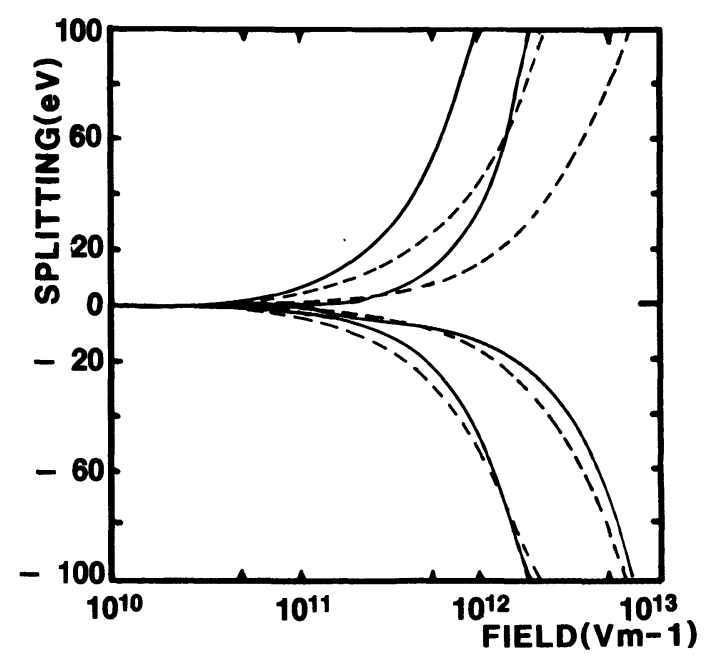

Fig. 1. - Energy splitting of the states $|\overline{4 i 0}\rangle$ of ${ }^{\mathrm{XVIII}} \mathrm{Ar}$ in $\mathrm{D}^{+} \mathrm{T}^{+}$(originating from $m=0$ ) versus electrostatic field value. Dashed lines correspond to homogeneous field, full lines to the first nearest neighbour approximation (1 NN). 
units the $V_{1}+V_{2}$ solutions are universal. There is a confusion in figure 2 of [2] which can be reconstructed from figures 1,3 and 5 of this work. Figures $2 a, b, c, d$, show the evolution of the eigenstates $|\overline{4 i 0}\rangle$ by their projections over the hydrogenic functions $|\overline{4 l 0}\rangle$. The dotted curves show the eigenvalues of the classical dipolar Hamiltonian only.

A $3 \times 3$ matrix deals with $|411\rangle,|421\rangle$ and $|431\rangle$ corresponding to $m=1$ and an identical one with $|41-1\rangle,|42-1\rangle,|43-1\rangle$ corresponding to $m=-1$. Figure 3 shows the energy splitting, each

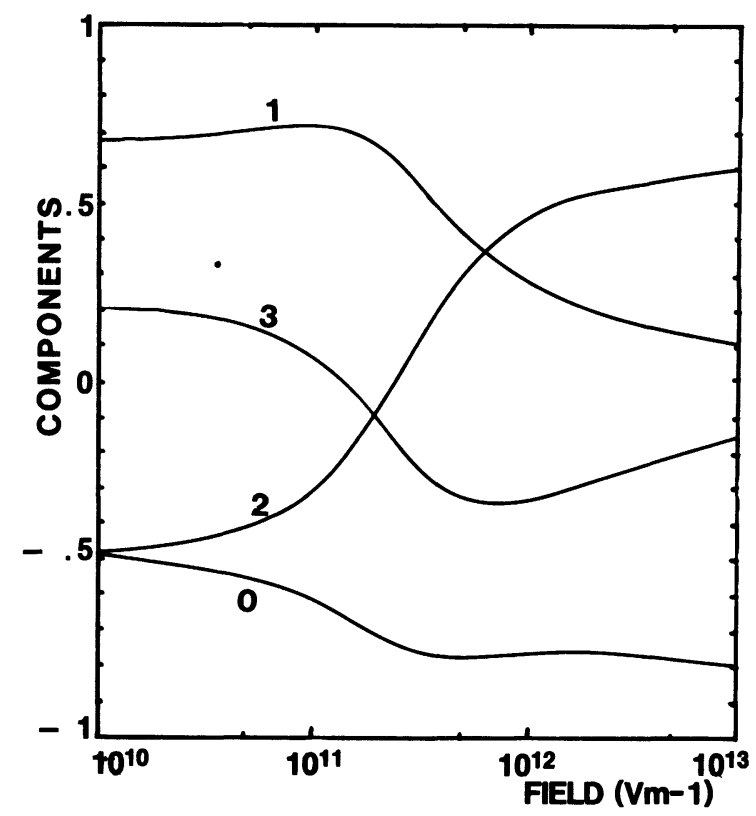

a)

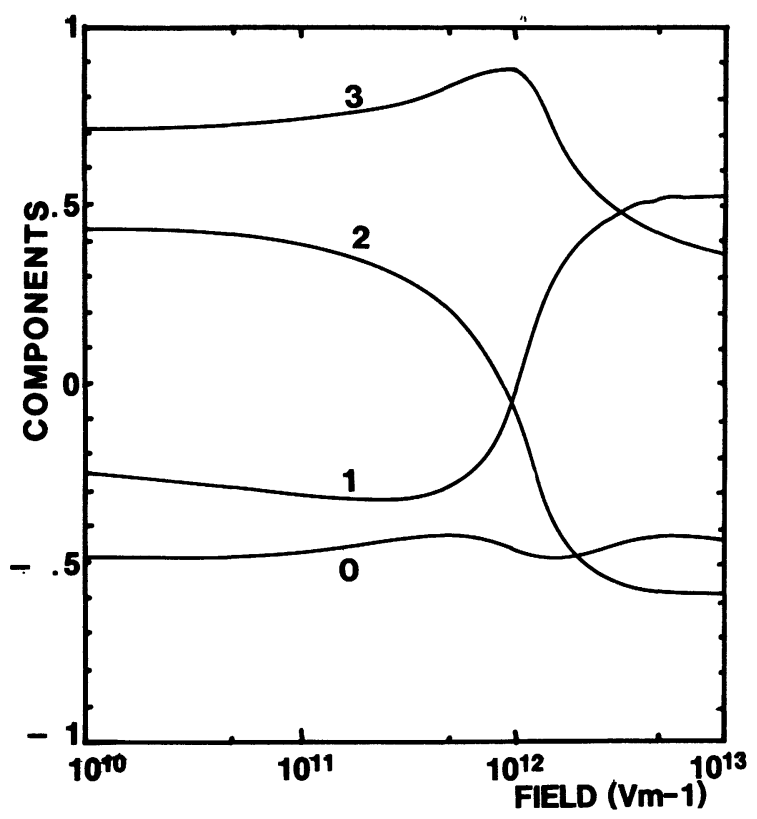

c) energy solution being doubly degenerate. Figures $4 \mathrm{a}$, $\mathrm{b}$, c, show the projections of the $|\overline{4 i \pm 1}\rangle$ over the corresponding $|4 l \pm 1\rangle$.

Two identical $2 \times 2$ matrices deal with the $(m=2)$ set $|422\rangle,|432\rangle$ and the $(m=-2)$ set $|42-2\rangle$, $|43-2\rangle$. The energy splitting is shown in figure 5 . Eventually, the states $|433\rangle$ and $|43-3\rangle$ remain unmodified $|\overline{413}\rangle=|433\rangle ;|\overline{41-3}\rangle=|43-3\rangle$, but there is a slight energy translation, also presented in figure 5. There is no possible dipolar emissivity from the states $m=2$ towards the final state $|\mathrm{f}\rangle=$

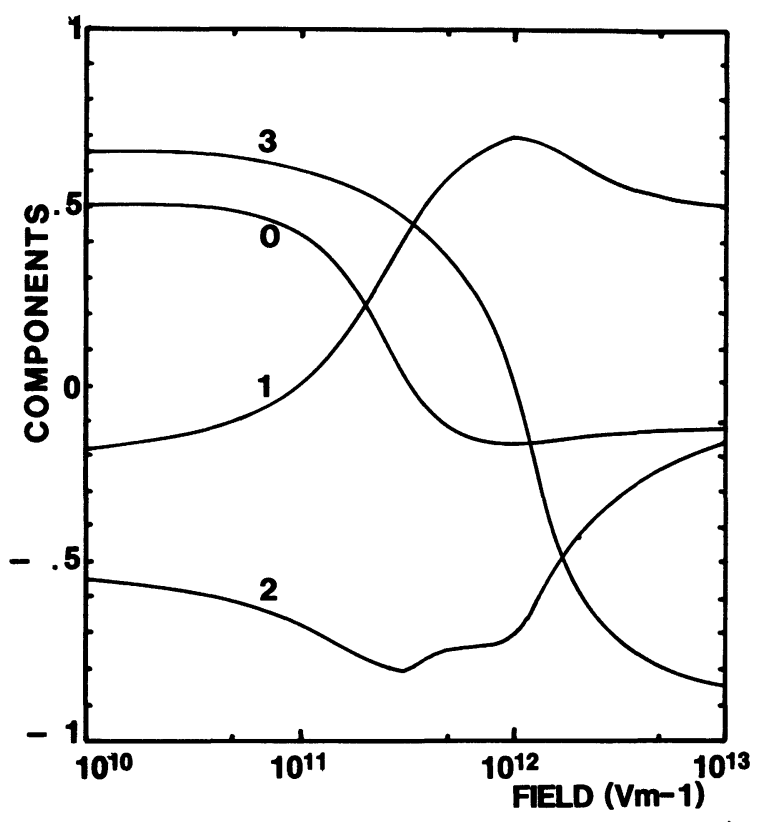

b)

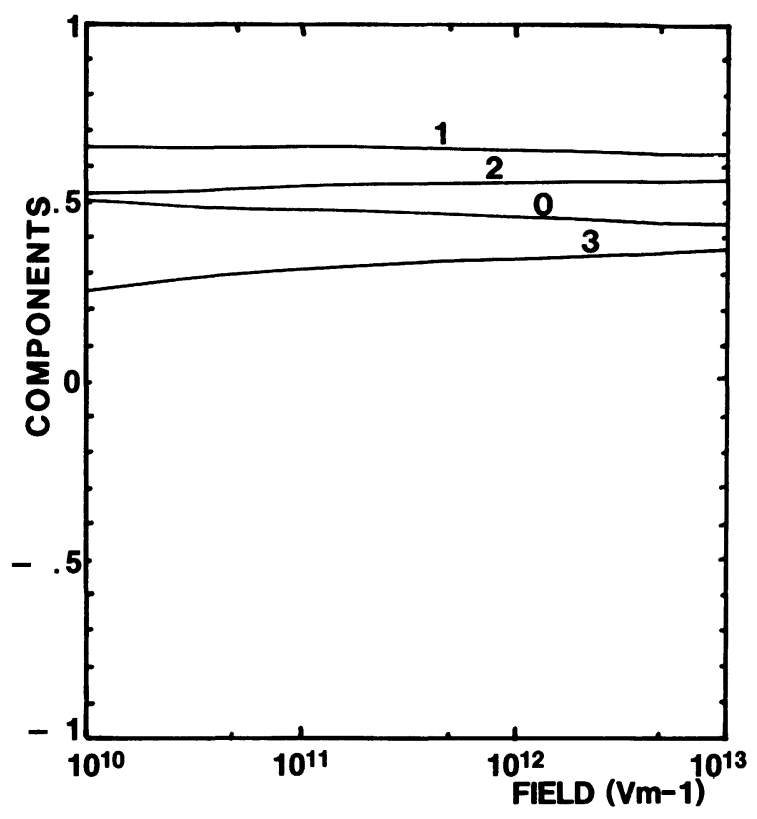

d)

Fig. 2. - Evolution versus electrostatic field of the states $|\overline{4 i 0}\rangle$ represented by their projections over $\{|4 l 0\rangle\}$ in $1 \mathrm{NN}$. The number identifies the state $l$ of projection $\langle\overline{4 i 0} \mid 4 l 0\rangle$. a) $i=1$; b) $i=2$; c) $i=3$; d) $i=4$. 


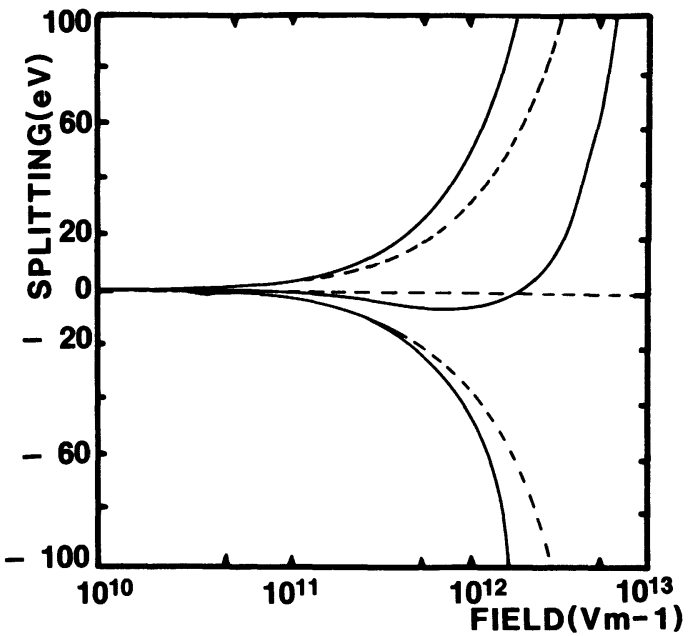

Fig. 3. - Energy splitting of the states $|\overline{4 i \pm 1}\rangle$ of ${ }^{\mathrm{xvIII}} \mathrm{Ar}$ in $\mathrm{D}^{+} \mathrm{T}^{+}$(originating from $m=1$ ) full : $1 \mathrm{NN}$, dashed : homogeneous field.

$|100\rangle$. So, the really meaningful energy behaviours are those presented in figure 1 and figure 3 . Let us review a few interesting features they present. The classical Stark symmetry is lost; but, up to high values of the field (a few $10^{11} \mathrm{~V} . \mathrm{m}^{-1}$ for argon), the difference is very slight. Afterwards the $1 \mathrm{NN}$ curves are shifted from the classical curves. For $|\overline{420}\rangle,|\overline{430}\rangle,|\overline{42 \pm 1}\rangle$, the shift is negative at low field and then positive. The opposite behaviour is true for $|\overline{440}\rangle$ and $|\overline{41 \pm 1}\rangle$. The eigenstates $\mid \overline{42 \pm 1}>$ also present the particularity of the appearance of one minimum $5.75 \mathrm{eV}$ lower than the previous line centre, for the field $8 \times 10^{11} \mathrm{~V} . \mathrm{m}^{-1}$. This range of field-values also corresponds to the maximal variation of the eigenstate projections versus field strength in figures 2 and 4 . The most negative eigenstates $|\overline{440}\rangle$ and $|\overline{43 \pm 1}\rangle$ are the least affected, and look very much like the homogeneous field solutions (which can be extrapolated by $F \rightarrow 0$ ). The most affected states are $|\overline{42 \pm 1}\rangle$ which present this energy minimum at $-5.75 \mathrm{eV}$. For the corresponding field value, the projections over the hydrogenic basis states change quite abruptly. The most positively shifted $|\overline{410}\rangle$ and $|\overline{41 \pm 1}\rangle$ suffer only a smooth crossing of their projections and the state $|\overline{420}\rangle$ presents an intermediary behaviour, its projection over $|410\rangle$ and $|420\rangle$ being transposed. So, the introduction of the further term of the field expansion has two kinds of consequences. First, the energy splitting is modified and its symmetry is broken.

Fig. 4. - Evolution of the states $|\overline{4 i \pm 1}\rangle$, represented by their projections over $\{|4 l \pm 1\rangle\}$ in $1 \mathrm{NN}$. The number $l$ identifies the state of projection $\langle\overline{4 i 1} \mid 4 l 1\rangle$. a) $i=1$; b) $i=2$; c) $i=3$.
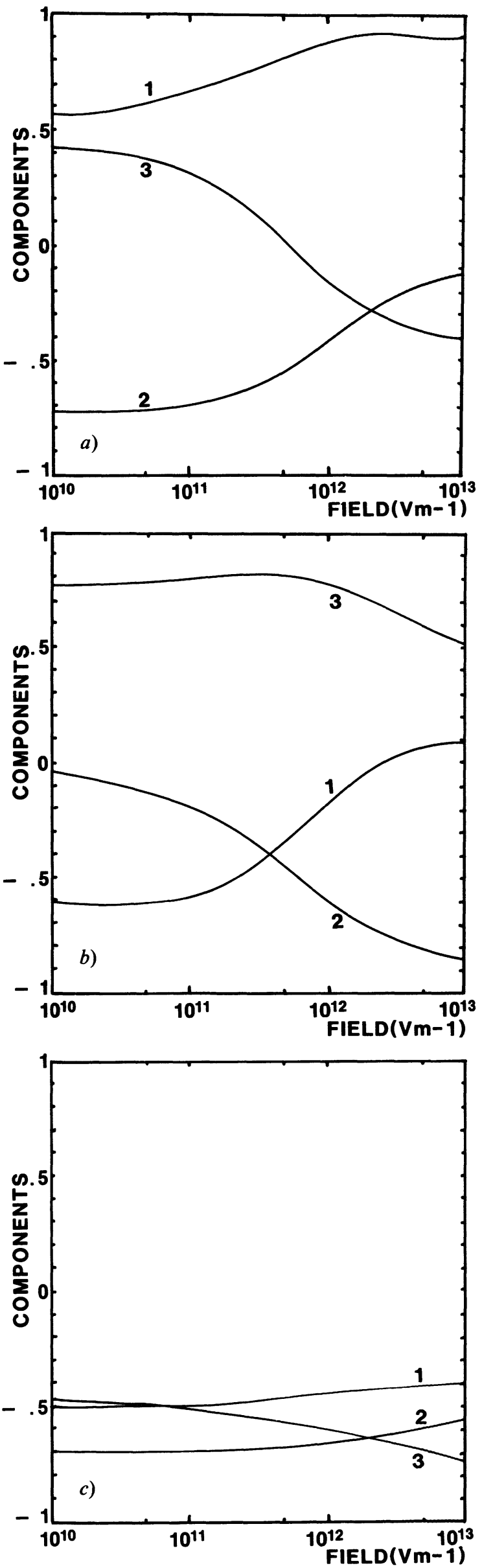


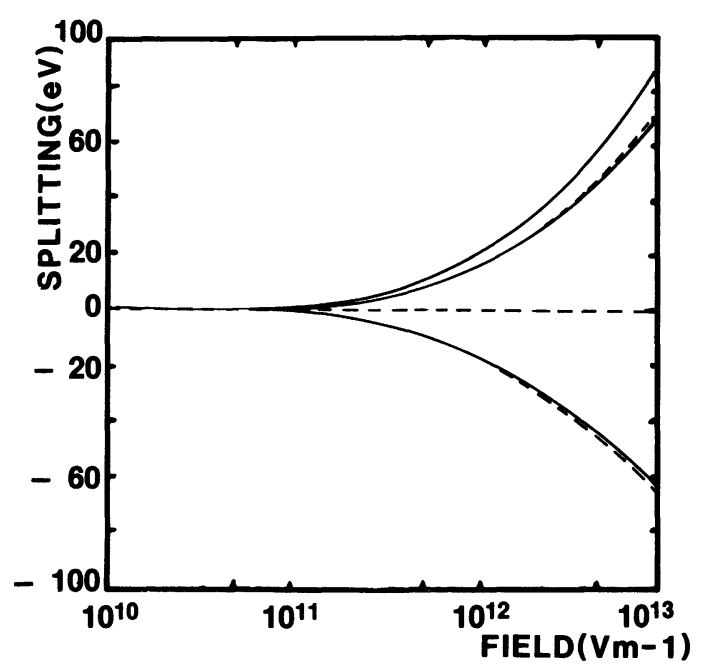

Fig. 5. - Energy splitting of the non-emissive states $|\overline{41 \pm 2}\rangle$. $|\overline{42 \pm 2}\rangle$ and $|\overline{41 \pm 3}\rangle$ full : $1 \mathrm{NN}$; dashed : homogeneous field.
But the second effect is even more important : the modification of eigenstate projections determines a drastic change of emission strength and then modifies the relative importance of the components.

\section{Calculation of the dipolar electric strength and} the quasi-static line profile.

In the line profile expression

$$
J(\omega)=\sum_{|j\rangle} \int \mathrm{d} F \mathfrak{T}(F)\|\langle j|\mathbf{d}| \mathbf{f}\rangle\|^{2} \delta\left(\omega-\omega_{j \mathrm{f}}\right)
$$

the dipolar term $\|\langle j|\mathbf{d}| \mathrm{f}\rangle\|^{2}$ can be easily determined since, as only the $l=1$ states can be coupled to $|100\rangle$, the radial part $\int r R_{n l} R_{10} r^{2} \mathrm{~d} r$ is common for all the eigenstates coming from the level $n$. For a given microfield $F$, the line profile is then

$$
J_{F}(\omega)=A\left\{\begin{array}{l}
\sum_{j=1}^{n}\|\langle n j 0|n| 0\rangle\|^{2}\|\langle n 10|\mathbf{d}| 100\rangle\|^{2} \delta\left(\omega-\omega_{|\overline{\mid n j 0}\rangle}\right) \\
+\sum_{j=1}^{n-1} \sum_{ \pm}\|\langle n j \pm 1|n| \pm 1\rangle\|^{2}\|\langle n 1 \pm 1|\mathbf{d}| 100\rangle\|^{2} \delta\left(\omega-\omega_{|\overline{\mid n j \pm 1}\rangle}\right)
\end{array}\right.
$$

Since

$$
\left\|\left\langle Y_{1}^{0}|\cos \theta| Y_{0}^{0}\right\rangle\right\|^{2}=\frac{1}{3}
$$

and

$$
\left\|\left\langle Y_{1}^{ \pm 1}|\sin \theta \cos \varphi| Y_{0}^{0}\right\rangle\right\|^{2}=\left\|\left\langle Y_{1}^{ \pm 1}|\sin \theta \sin \varphi| Y_{0}^{0}\right\rangle\right\|^{2}=\frac{1}{6}
$$

the line profile for a given field is then, taking into account the $m= \pm 1$ degeneracy

$$
J_{F}(\omega)=\sum_{j=1}^{n}\|\langle\overline{n j 0}|r| 0\rangle\|^{2} \delta\left(\omega-\omega_{|\overline{\mid n j 0}\rangle}\right)+2 \sum_{j=1}^{n-1}\|\langle\overline{n j 1}|r| 1\rangle\|^{2} \delta\left(\omega-\omega_{\overline{\mid n j i}\rangle}\right)
$$

this line profile being the mere addition of $(2 n-1)$ discrete frequencies.

This treatment does not take into account the continuum part of the solutions of the Hamiltonian. A correction can be added for this configuration interaction [6] as a perturbation. But the only direct incorporation is the calculation of the dipolar strength, the energy being the variable. With the dipolar term only, the calculation is possible for each value of $F$ [7] but the integration over $F$ would be quite costly in computer time.

The projections of $|\overline{4 j 0}\rangle$ and $|\overline{4 j \pm 1}\rangle$ over $|410\rangle$ and $|41 \pm 1\rangle$ are shown in figure 2 and figure 4 . One can check how important the contribution of the term $\Delta \mathrm{H}_{2}$ of Stark perturbation is by listing the modification of the emitting strength,

$$
\text { for } \begin{array}{rlll}
\|\langle 410 \mid \overline{410}\rangle\|^{2} & \text { from } & 0.5 & \text { to } 0.05 \\
\|\langle 410 \mid \overline{420}\rangle\|^{2} & 0.04 & 0.5 \text { via } 0 \\
\|\langle 410 \mid \overline{430}\rangle\|^{2} & 0.06 & 0.25 \text { via } 0 \\
\|\langle 410 \mid \overline{440}\rangle\|^{2} & 0.4 & 0.4 \\
\|\langle 411 \mid \overline{411}\rangle\|^{2} & 0.35 & 0.8 \\
\|\langle 411 \mid \overline{421}\rangle\|^{2} & 0.35 & 0.02 \text { via } 0 \\
\|\langle 411 \mid \overline{431}\rangle\|^{2} & 0.25 & 0.2
\end{array}
$$

Only the most negative states, $|\overline{440}\rangle$ and $|\overline{43 \pm 1}\rangle$ keep a quasi-constant emitting strength. The central states $|\overline{420}\rangle$ and $|\overline{430}\rangle$ gain a more important contribution at the expense of $|\overline{410}\rangle$. The central peak $|\overline{42-1}\rangle$ loses most of its power to $|\overline{41 \pm 1}\rangle$. 
This is to be compared to the fixed dipolar strength of $a$ priori parabolic states, with no $F$ dependent evolution, such as the $|\mathrm{i}\rangle$ described in [8], Appendix, with the same limitation to the $n=4$ subspace.

To represent the trace of the density operator over the external conditions - i.e. the field value - we make the integration

$$
J(\omega)=\int \mathrm{d} F \mathfrak{T}(F) J_{F}(\omega) \delta\left(\omega-\omega_{j(F)}\right)
$$

using the transformation

$$
\delta\left(\omega-\omega_{j(F)}\right) \rightarrow \sum_{k}\left|\left(\frac{\mathrm{d} \omega}{\mathrm{d} F}\right)^{-1}\right| \delta\left(F-F_{k}\right)
$$

provided that $F_{k}$ is the solution of $\omega\left(F_{k}\right)=\omega$. The parameter $k$ distinguishes the solutions of this equation when $\omega_{j(F)}$ is non-monotonous, that is for $|\overline{42-1}\rangle$. The line profile then becomes :

$$
J(\omega)=\int \mathrm{d} F \mathfrak{T}(F)\left\{\begin{array}{c}
\sum_{j=1}^{n} \sum_{k}\|\langle\overline{n j 0}|n| 0\rangle\|^{2}\left|\frac{\mathrm{d} \omega_{|\overline{n j 0}\rangle}}{\mathrm{d} F}\right|^{-1} \delta\left(F-F_{k|\overline{n j 0}\rangle}\right) \\
+2 \sum_{j=1}^{n-1} \sum_{k}\|\langle\overline{n j 1}|n| 1\rangle\|^{2}\left|\frac{\mathrm{d} \omega_{|\overline{n j 1}\rangle}}{\mathrm{d} F}\right|^{-1} \delta\left(F-F_{k \mid \overline{n j 1}}\right)
\end{array}\right.
$$

One can see here that the advantage of choosing $J(\omega)$ instead of the more significant $P(\omega)$ is the simplification of the density operator which is supposed factorizable into the product of the external condition $F$ with the quantum response $|j\rangle$. This allows all permutations of the integrations over $F$ and $|j\rangle$.

Since the assumption $\langle r\rangle \leqslant R_{\mathrm{p}}$ determines a limitation $F \leqslant F_{\mathrm{m}}$, the integration for much higher field values has practically no physical meaning. But the field distribution has no cut off. For XVIII argon in $\mathrm{D}^{+} \mathrm{T}^{+}$, the peak of the distribution function [5] reaches 0.35 for $F=1.5 F_{0}$. So we must limit the density to lower values such that the fields above $F_{\mathrm{m}}$ have a very low probability and thus a very small energy contribution to the line profile. If we accept a maximal probability $10^{-4}$ for $F_{\mathrm{m}}$, then $F_{\mathrm{m}} \geqslant 25 F_{0}$ and $N_{\mathrm{e}} \leqslant 2 \times 10^{22} \mathrm{~cm}^{-3}$. For the maximal probabi-

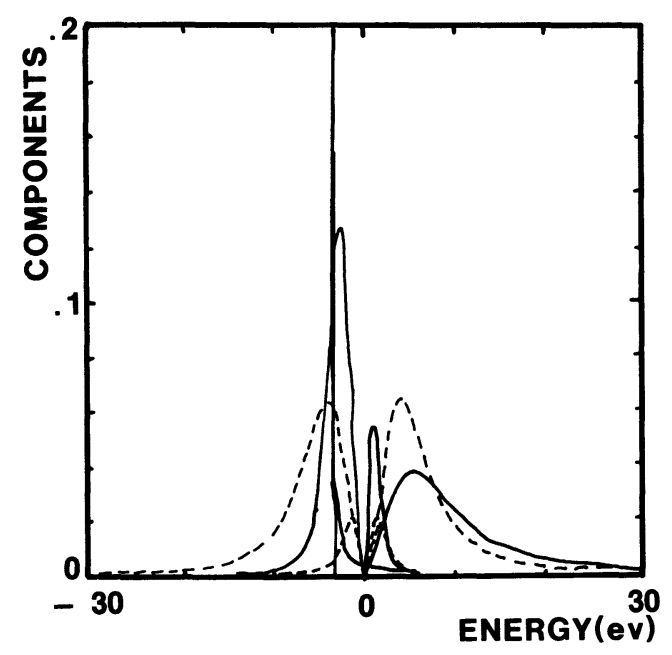

Fig. 6. - Line components $|\overline{4 i 0}\rangle$ originating from $m=0$ for electron density $7 \times 10^{22} \mathrm{~cm}^{-3}$. The intensity unit is arbitrary but common for homogeneous field (dashed) and inhomogeneous field (full line). lity $2 \times 10^{-3}$ then $N_{\mathrm{e}} \leqslant 6 \times 10^{22} \mathrm{~cm}^{-3}$ and for $5 \times 10^{-3}, N_{\mathrm{e}} \leqslant 10^{23} \mathrm{~cm}^{-3}$.

To be on the conservative, we present our calculation of argon Lyman $\gamma$ for $N_{\mathrm{e}}=7 \times 10^{22} \mathrm{~cm}^{-3}$ at a temperature $k T=450 \mathrm{eV}$ (Hooper's parameter is then 0.2 ).

Figure 6 shows the components for $m=0$ and figure 7 for $m=1$. The intensity units are arbitrary, but the same for these two figures.

For the homogeneous $F$ field model (HFM), the $|\overline{42 \pm 1}\rangle$ components are unshifted Dirac distributions. Since their emission power equals that of $\mid \overline{41 \pm 1}$ or $|\overline{43 \pm 1}\rangle$, by integration over $F$, we determine the central peak profile of homogeneous field Lyman $\gamma$ lines [11], thus escaping from the numerical problem of the normalization of these Dirac distributions.

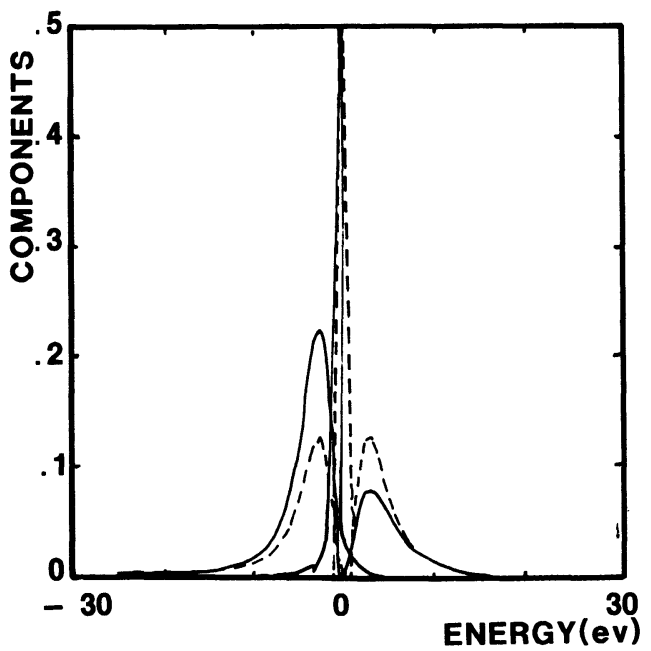

Fig. 7. - Line components $|\overline{4 i \pm 1}\rangle$ originating from $m= \pm 1$ with the same as previous arbitrary unit. Density is $7 \times 10^{22} \mathrm{~cm}^{-3}$. The homogeneous field originated component is approximated here by a triangle (for this figure only and not in the calculation). 
For the inhomogeneous $F$ field, the corresponding $|\overline{42 \pm 1}\rangle$ presents a non-monotonous shift resulting in a shifted sharp peak - due to the fact that the eigen-energy of $|\overline{42 \pm 1}\rangle$ is non-monotonous. This peak, of course, can only be distinguished when the microfield probability is not too low for the field value of the energy extremum.

The addition of all components into the quasistatic line profile is shown in figure 8 at high density to demonstrate this behaviour of $|\overline{42 \pm 1}\rangle$.

Figure 9 presents this profile for a low density where differences between homogeneous and first neighbour fields are of a lesser importance - except at the very centre.

At higher densities the central region of the total line - one unshifted peak for homogeneous field or two peaks in $1 \mathrm{NN}$ - is the most sensitive part of the line to the effect of any other broadening phenomena. Electron Stark effect and thermal Doppler and

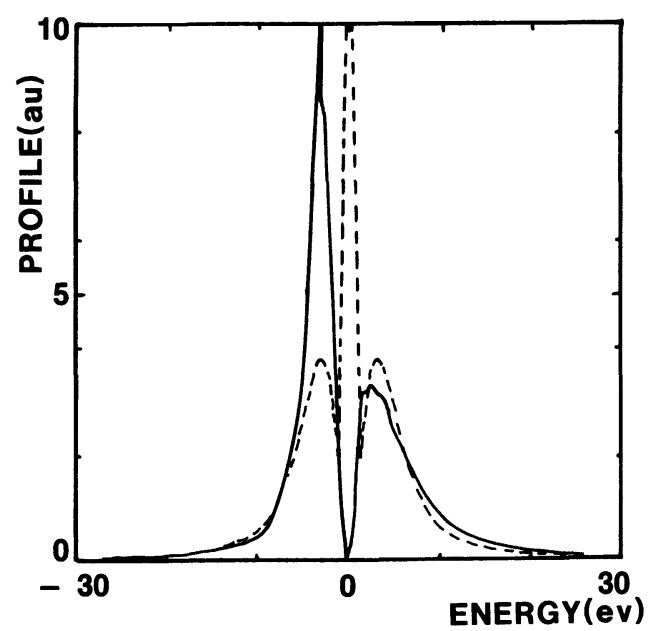

a)

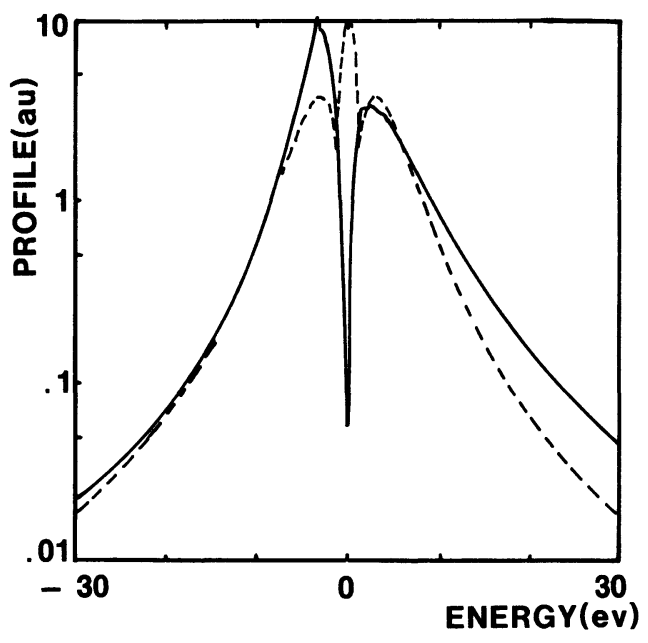

b)

Fig. 8. - Total quasi-static line profile for $7 \times 10^{22} \mathrm{~cm}^{-3}$ in $1 \mathrm{NN}$ approximation. The line presented in linear a) and semi-log b) representations.

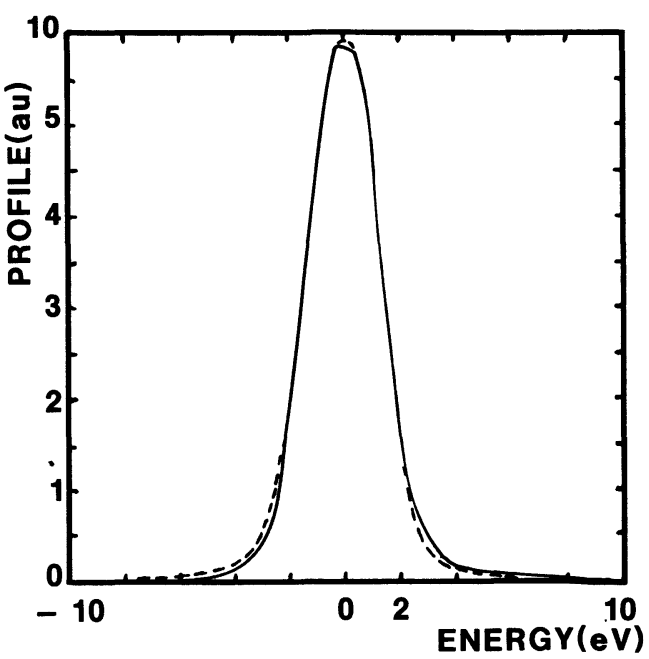

a)

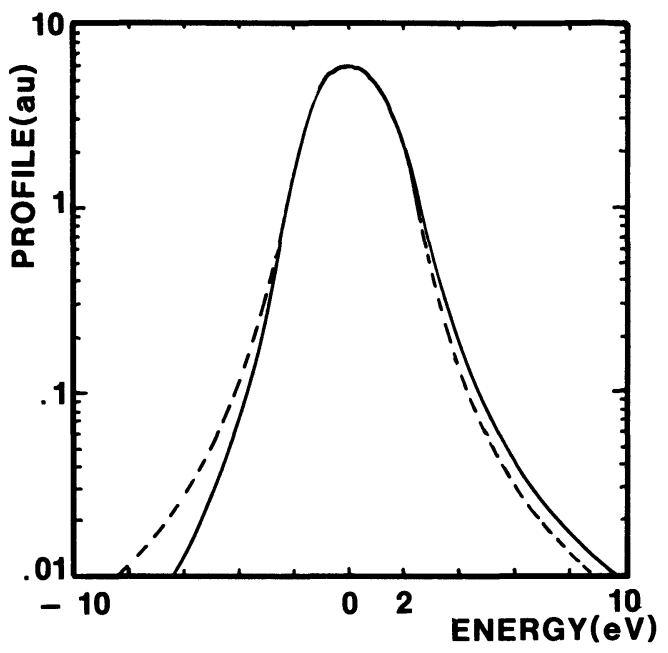

b)

Fig. 9. - Same representation of quasi-static line profile at the lower density $5 \times 10^{21} \mathrm{~cm}^{-3}$, including Voigt convolution.

ion dynamics effects result in a Voigt convolution of the mere quasi-static profile :

$$
J(\omega)=\int \mathrm{d} F \mathfrak{T}(F) J_{F}(\omega) V\left(\omega-\omega_{\mathrm{i} F(F)} .\right.
$$

The Lorentzian-like electronic impact parameter is routinely calculated from [1]

$$
\begin{aligned}
& \hbar \Delta \omega \sim \frac{N n^{4}}{Z^{2} \sqrt{k T}} 5.42 \times 10^{-29} \mid \log \frac{Z(k T)^{3 / 2}}{N n^{2}} \times \\
& \times 2.97 \times 10^{-17}-\frac{1}{8} \mid .
\end{aligned}
$$

$N$ being in $\mathrm{m}^{-3}, k T$ in $\mathrm{eV}$ and $\hbar \Delta \omega$ also in $\mathrm{eV}$.

A more recent expression can be found in [9] but this last work fits lower density experiments better than laser plasmas. 
The Gaussian-like width corresponding to emitter and perturber thermal effects is calculated from the quadratic summation $\Delta W^{2}=\Delta W_{\mathrm{D}}^{2}+\Delta W_{\mathrm{ID}}^{2}$ of the usual Doppler width $W_{\mathbf{D}}$ and the ion dynamics with [10] :

$$
W_{\mathrm{ID}}=2 h\left(1.33 \pi N_{\mathrm{e}}\right)^{+1 / 3}\left(\frac{3 b k T}{M_{i}}\right)^{1 / 2}
$$

which dominates $(2.17 \mathrm{eV}$ against $0.62 \mathrm{eV})$, Griem's constant $b$ being extrapolated from [10] to the value 1.2 .

The three parameters $\hbar \Delta \omega, \Delta \omega_{\mathrm{D}}$ and $\Delta W_{\mathrm{ID}}$ are considered as constants for the complete convolution.

We present in figure 10 this convoluted profile corresponding to figure 8 . One can check that the general shape is little affected (in absence of all reabsorbing treatment, of course) by this convolution. So we can rely upon a very simple approximation of

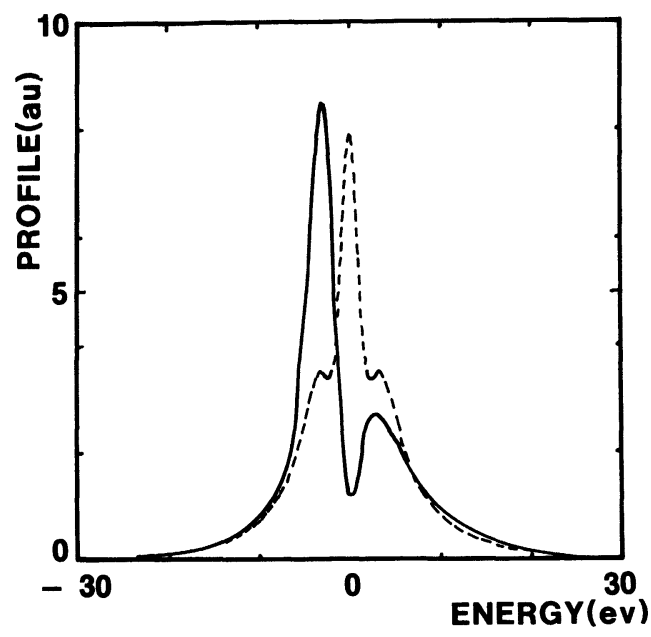

a)

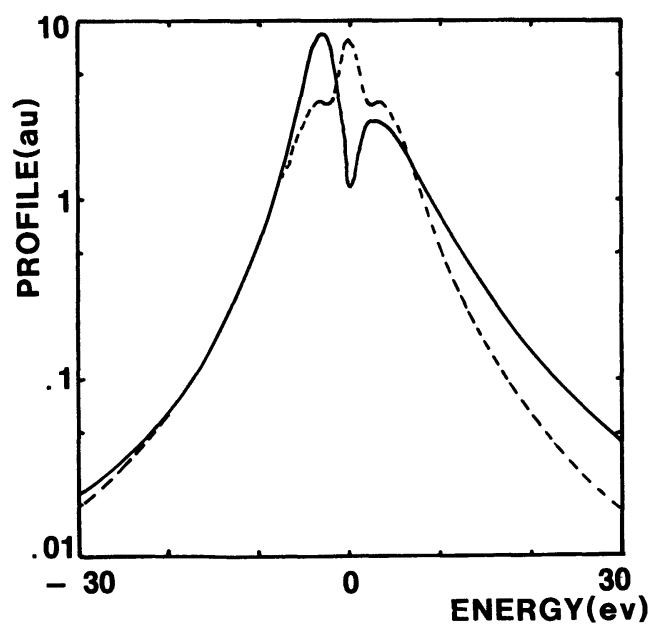

b)

Fig. 10. - Total line profile for $7 \times 10^{22} \mathrm{~cm}^{-3}$, corresponding to figure 8 but including Voigt profile of electronic Stark, Doppler and ion dynamics broadenings, linear a); semi-log b). electron impact width for density estimations - and justify the efforts towards a better description of the quasi-static phenomena by the fact they are the most significant.

\section{Discussion.}

In this paper, we have expanded the field expression, using the single first neighbour approximation, to its first Taylor terms, dipolar and quadrupolar. But, since the real plasma is a much more than three-body problem, the field determination comes from a statistical calculation using all the neighbouring ions in a correlation hierarchy. So there is something artificial in the use of a single perturber quantum development, associated to a statistical expression of the field itself, instead of real perturber positions, but not more artificial than the usual assumption of homogeneous fields.

For large values of the field - the scale being the Holtsmark value $F_{0}$ - the most probable situation is that the field is nearly almost created by a single very close perturber. If two near ions were to create this field, since their directions should not be very different, replacing both of them by a single closer one would only result in a factor, the exceptional maximum of which would be 2 - which is acceptable enough. But, for low values of the field, the most probable situation corresponds to the destructive addition of a few comparable fields. In fact, a preponderant supremacy of a first neighbour creating a weak field would mean it should be further from the emitter than the mean distance between ions - which is absurd. Anyway, the first nearest-neighbour approximation does not require the total plasma configuration to be reduced to a single perturber. It remains valid whenever the main differences from spherical symmetry may be attributed to a single perturber.

So the previous relation between the field and its first moments as calculated from $1 \mathrm{NN}$ should be corrected, since it is only a limit for high fields. To test whether such an improvement is of real importance, we used the purely mathematical factor th $\left(F / \alpha F_{0}\right)$ inserted in the gradient effect [11], $F_{0}$ being the Holtsmark field. This is smoother than a mere cut-off of the quadrupolar effect for fields lower than a few $F_{0}$. The new Hamiltonian is then :

$$
\begin{aligned}
H=H_{0}+\Delta H_{1}+\Delta H_{2} \text { th }\left(\frac{F}{\alpha F_{0}}\right) \\
=H_{0}-q F r \cos \theta-\operatorname{th}\left(\frac{F}{\alpha F_{0}}\right) \times \\
\quad \times q F^{3 / 2} \sqrt{\frac{4 \pi \varepsilon_{0}}{Z_{\mathrm{p}} q}} r^{2} \frac{3 \cos ^{2} \theta-1}{2} .
\end{aligned}
$$

Results for energy splittings as well as for dipolar strengths prove that even for $|\overline{430}\rangle$ and $|42 \pm 1\rangle$, which present the most chaotic behaviour, the diffe- 


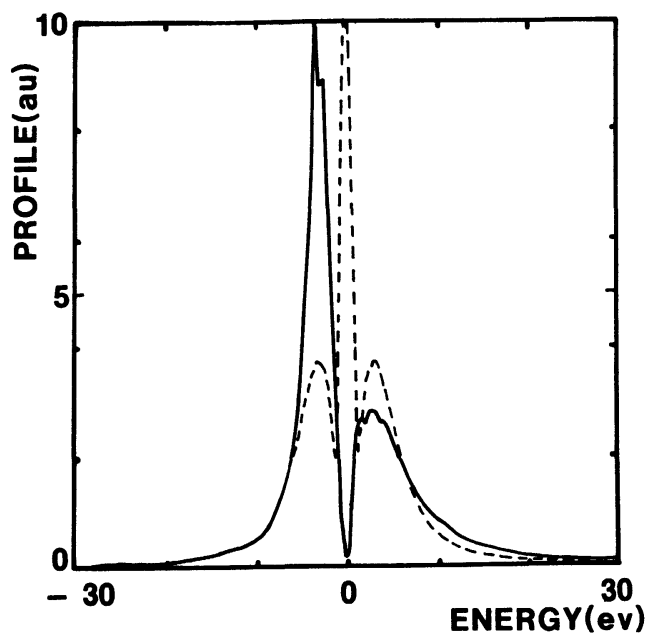

a)

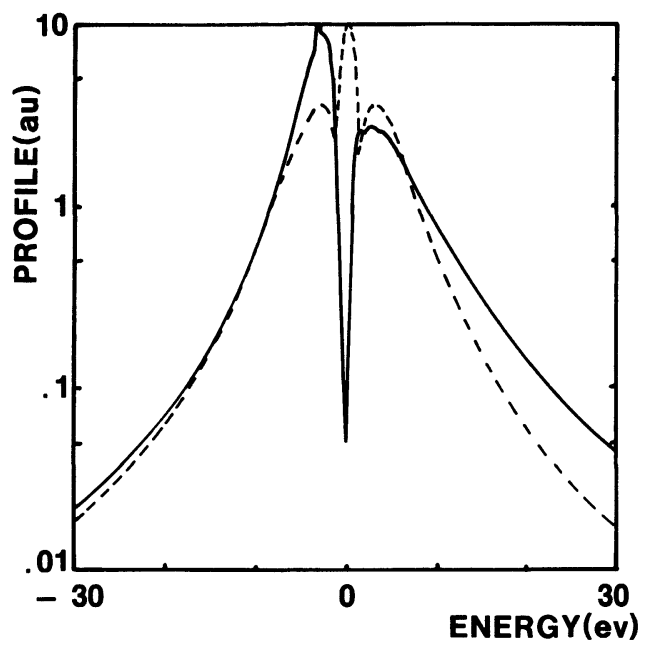

b)

Fig. 11. - Total quasi-static line profile for $7 \times 10^{22} \mathrm{~cm}^{-3}$ corresponding to figure $8 \mathrm{a})$, b) but including the limiting factor th $\left(F / F_{0}\right)$.

rence created by the test factor th $\left(\frac{F}{\alpha F_{0}}\right)$ is noticeable but not very important. We present the line shapes we obtained with this factor th $\left(F / \alpha F_{0}\right)$. Figures 11a and $b$ with $\alpha=1$. Figures $12 \mathrm{a}$ and $\mathrm{b}$ show the effects of Doppler and impacts with the same parameters $\hbar \Delta \omega, \Delta W_{\mathrm{D}}$ and $\Delta W_{\mathrm{ID}}$ as in figures $10 \mathrm{a}$ and $\mathrm{b}$.

We present in figures $13 \mathrm{a}$ and $\mathrm{b}$ the calculation using the factor th $\left(\frac{F}{\alpha F_{0}}\right)$ for the values $1,2,3$ and 5 of $\alpha$. We test that the wings of the line remain nearly unmodified. But the computation of the line core presents a numerical problem.

For all eigenstates, the derivative factor $\left(\frac{\mathrm{d} \omega_{|n j i\rangle}}{\frac{\mathrm{d} F}{421}}\right)$ is low for low field values. It remains zero for $|\overline{421}\rangle$ in the HFM calculation, but since in HFM all dipolar

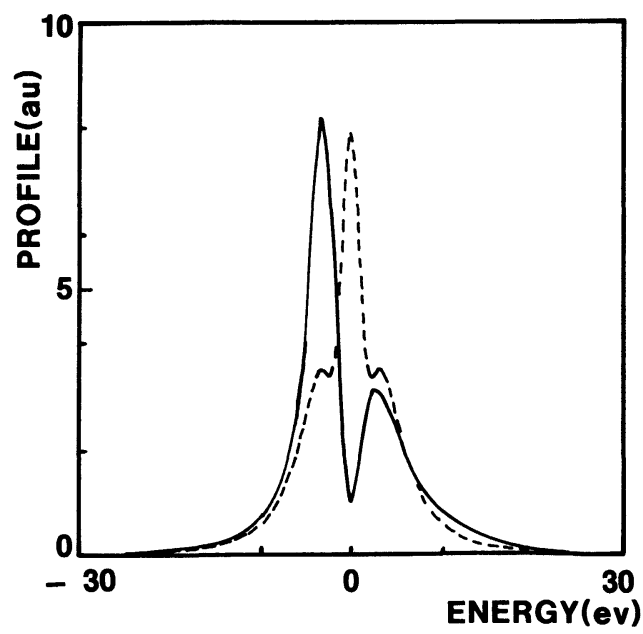

a)

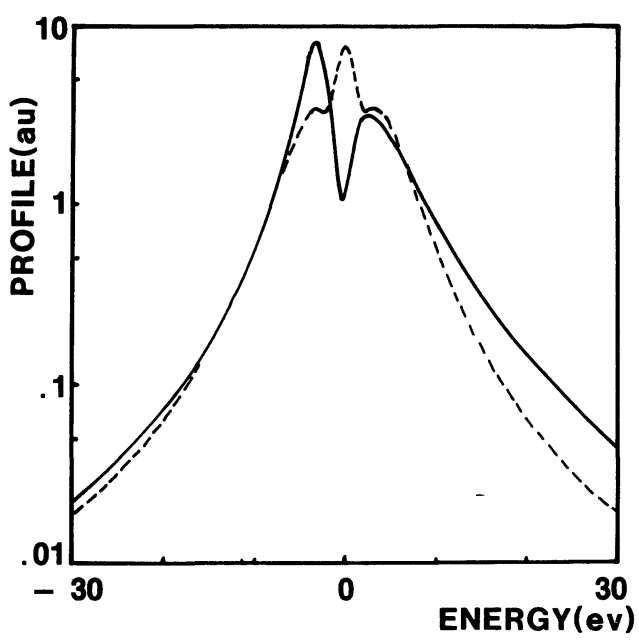

b)

Fig. 12. - Total line profile for $7 \times 10^{22} \mathrm{~cm}^{-3}$ with factor th $\left(F / F_{0}\right)$ and including Voigt convolution of electronic Stark, Doppler and ion dynamics broadening.

strengths remain constant, the normalization of $|\overline{421}\rangle$ delta peak from the energy of $|\overline{411}\rangle$ is easy.

In $1 \mathrm{NN}$ and th-modified $1 \mathrm{NN}$, there are two regions where $\left(\frac{\mathrm{d} \omega_{|\overline{421}\rangle}}{\mathrm{d} F}\right)$ nears zero : for the low values of $F$ and around a value $F_{\mathrm{M}}$ for which $|\overline{421}\rangle$ energy reaches a minimum. Both regions give a peak by integration over the microfield distribution.

For pure $1 \mathrm{NN}$, or for $\alpha$ lower than $2.5, F_{\mathrm{M}}$ is located in a region of high field probability. Then the peak corresponding to $W=W\left(F_{\mathrm{M}}\right)$ is important, and the peak corresponding to $W=0$ is not.

For $\alpha$ higher than $2.5, F_{\mathrm{M}}$ outranges the region of high probability of the field. Then the two peaks are of comparable importance, but their proper balance is uncertain with our algorithm. The reason is that the presence of two peaks makes it impossible to use a 


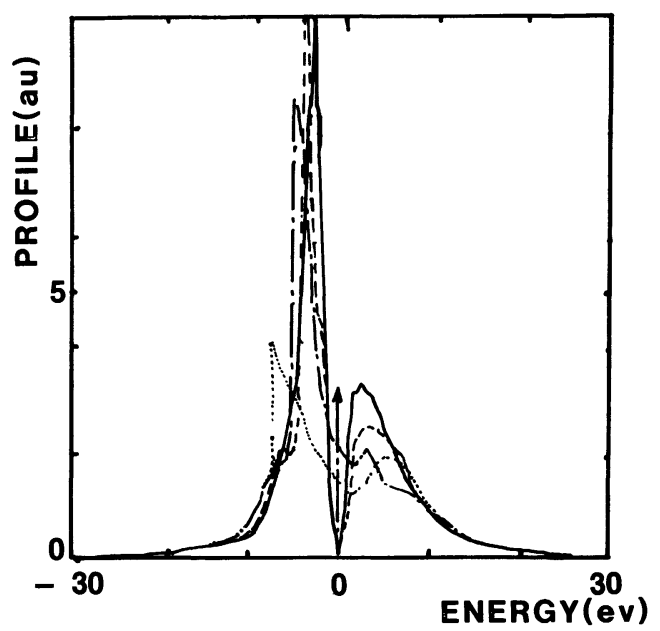

a)

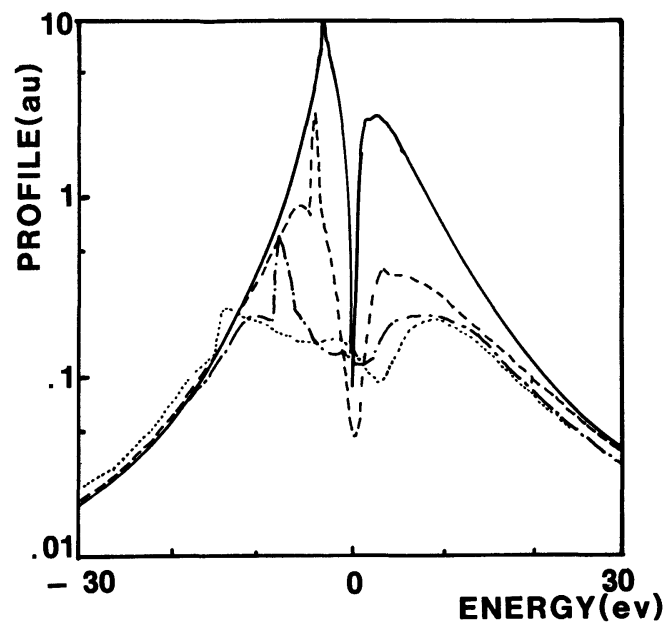

b)

Fig. 13. - Comparison between the line profiles corresponding to the values $1,2,3$ and 5 for $\alpha$ in th $\left(F / \alpha F_{0}\right)$. Full line represents $\alpha=1$, dashed line is for $\alpha=2$, dashed-dotted line for $\alpha=3$ and dotted line for $\alpha=5$.

These figures correspond to figures $8 \mathrm{a}$ ), b) and 11a), b).

calibration originating from the single peak of homogeneous field. Then the energy must be calculated directly from the expression $J(\omega)$ using $|\mathrm{d} \omega / \mathrm{d} F|^{-1}$. Since this factor tends to infinity, it is very sensitive to the chosen values of the $F$ steps. And two calculations using slightly different values for $F$ can correspond to nearly different ratios of the two peaks. So we prefer to point out the problem of this normalization.

Figures 13a and $b$ show the line shapes corresponding to the values $\alpha=1,2,3$ and 5. For the last two values at least, the numerical uncalibration of the central peak is symbolized by an arrow. The uncertainty corresponds to a region

$$
\begin{aligned}
& -2 \leqslant \Delta W \leqslant+2 \mathrm{eV} \text { for } \alpha=1 \text { or } 2 \\
& -4 \leqslant \Delta W \leqslant+2 \mathrm{eV} \text { for } \alpha=3 \\
& -7 \leqslant \Delta W \leqslant+2 \mathrm{eV} \text { for } \alpha=5 .
\end{aligned}
$$

This domain must be compared to the spectral precision of experimental results. One must also keep in mind that this is the region the most affected by reabsorption effects.

We can also verify that the line wings remain untouched by adjusting the normalization parameters of each line so that all the corresponding wings get superposed.

So we think that the comparison of the $1 \mathrm{NN}$ with th $\left(F / F_{0}\right)$ improved $1 \mathrm{NN}-$ or even up to th $\left(\frac{F}{2.5 F_{0}}\right)$ improved $1 \mathrm{NN}-$ is a validation of the compatibility between $1 \mathrm{NN}$ Hamiltonian and the microfield distribution. A better justification would be the comparison of $1 \mathrm{NN}$ gradient against the results of Monte-Carlo statistical simulation of the perturbers' positions.

Since the proper use of an expression including both the field value and its moments [12] $P\left(\mathbf{F}, \frac{\partial \mathbf{F}}{\partial \mathbf{r}}\right)$ should correctly lead to a multiple variable integration, we consider the use of $1 \mathrm{NN}$ with the simple microfield probability $P(F)$ as an improvement over HFM.

\section{Conclusion.}

The main points of this work are :

- First, the quasi-static perturbation is treated in the nearest neighbour approximation, with the introduction of the first non-linear Hamiltonian terms. This part of the calculation is essential since it directly governs the profile of the non-absorbed line wings.

- Then to these fundamental solutions corresponding to large times, it is easy to add the effects of electron impacts and thermal Doppler broadening and even the instrumental function.

To compare our results to previous calculations of dipolar perturbation only, we verified the H.W.H.M. is not really different - at least in the laser plasma temperature and density range of a few $10^{2} \mathrm{eV}$ and $10^{21}-10^{23} \mathrm{~cm}^{-3}$. But the shape is modified, and no longer symmetrical, even after the further Lorentzian and Gaussian treatments.

Let us now consider the impact on the density diagnosis of plasmas. For an isolated line, such as argon Lyman $\gamma$ (presented here) or He-like argon $\left(1 \mathrm{~s} 4 \mathrm{p}-1 \mathrm{~s}^{2}\right)$ one tries to reproduce the experimental curves by adjusting the values of the parameters $\left(T, N_{\mathrm{e}}\right)$.

The couple of parameters $\left(T, N_{\mathrm{e}}\right)$ which corresponds to the best coincidence between the calculation and the spectroscopic data is then taken as representative of the real plasma X-ray emission.

The coincidence of a whole theoretical line profile, including wing asymmetries, with the spectroscopic data provides a far better test of the possibility to represent the plasma emission by a single couple 
$\left(T, N_{\mathrm{e}}\right)$ than the mere comparison of measured and calculated H.W.H.M.

But a real line is very seldom isolated from the other nearest lines, except for the resonance transitions from level 2 to level 1, which are subject to reabsorption. Many other lines, such as argon $3 \mathrm{p}-1 \mathrm{~s}$ and $1 \mathrm{~s} 4 \mathrm{p}-1 \mathrm{~s}^{2}$ and $1 \mathrm{~s} 5 \mathrm{p}-1 \mathrm{~s}^{2}$ are merged together. And anyway, all lines are superposed on a continuum and the main lines are flanked by satellites.

To use the spectroscopic profile of a non-isolated line, one has to extract its original shape from the superposition of profiles and this requires the know- ledge of the theoretical asymmetry of each of the lines which are merged. And to use the energy ratio of satellites, one needs to subtract the wing of the principal line. Computer programs have been presented for processing experimental data into the numerical separation of merged lines [13], using mainly a modelization profile (Lorentzian or Gaussian).

Our present work which points out the danger of reconstitution of merged lines by symmetry only, presents an alternative by computing the main line wing asymmetry and only afterwards discriminating satellites in the experimental data.

\section{References}

[1] GRIEM, H., Spectral line broadening in plasmas (Acad. Press) 1974.

[2] Lambert, D., Optics Commun. 34 (1980) 209. The sign of $|\overline{420}\rangle$ in figure 2 is wrong and must be corrected from this paper.

[3] Griem, H., Plasma spectroscopy (McGraw Hill) 1964.

[4] HoOper, C. F., Phys. Rev. 149 (1966) 77 ; 165 (1968) $215 ; 169$ (1968) 193.

O'brien, J. T. and HoOper, C. F., Phys. Rev. A 5 (1972) 867.

Tighe, J. R. and Hooper, C. F., Phys. Rev. A 17 (1978) 40.

[5] Held, B., Private Communication.

[6] Tighe, R. J., Ph. D. Thesis 1977 (Univ. of Florida).
[7] Bethe, H. and SAlPeter, E., Quantum Mechanics of one- and two-electron atoms (Springer Verlag) 1957.

Luc, E., J. Phys. B 13 (1980) 1767, 1769-1790.

[8] BACON, M. E., J.Q.S.R.T. 13 (1973) 1161 ; J.Q.S.R.T. 17 (1977) 501.

[9] Griem, H., Phys. Rev. A 27 (1983) 2566.

[10] Cauble, R. and Griem, H., Phys. Rev. A 27 (1983) 3187.

[11] LAMBeRT, D., 5e ICSLS Berlin 1980, 14 ECLIM Palaiseau 1980

[12] Sholin, G. V., Optics Spectros. 26 (1969) 275.

Demura, A. V. and Sholin, G. V., J.Q.S.R.T. 15 (1975) 881

[13] Lambert, D., Sauneuf, R., Tenaud, J. P., 14e ECliM, Palaiseau 1980. 